\title{
Repères
}

\section{Ukraine : l'enseignement supérieur et ses enjeux actuels}

\section{Marina Sacilotto-Vaylenko}

\section{OpenEdition}

\section{Journals}

Édition électronique

URL : http://journals.openedition.org/ries/731

DOI : $10.4000 /$ ries.731

ISSN : 2261-4265

Éditeur

Centre international d'études pédagogiques

Édition imprimée

Date de publication : 1 septembre 2009

Pagination : 148-154

ISSN : 1254-4590

Référence électronique

Marina Sacilotto-Vaylenko, « Ukraine : l'enseignement supérieur et ses enjeux actuels », Revue internationale d'éducation de Sèvres [En ligne], 51 | septembre 2009, mis en ligne le 01 septembre 2012, consulté le 20 avril 2019. URL : http://journals.openedition.org/ries/731 ; DOI : 10.4000/ries.731

Ce document a été généré automatiquement le 20 avril 2019.

(C) Tous droits réservés 


\title{
Repères
}

\section{Ukraine : l'enseignement supérieur et ses enjeux actuels}

\author{
Marina Sacilotto-Vaylenko
}

\section{Démocratisation et européanisation}

1 Depuis la proclamation de son indépendance en 1991, l'Ukraine a commencé à construire sa politique éducative et son système d'enseignement supérieur. Hérités de l'époque soviétique, la centralisation, l'intervention étatique à tous les niveaux (gestion du système, planification des cadres, choix des contenus et des méthodes, etc.) et une forte idéologisation de l'éducation étaient considérés comme les principaux obstacles au développement d'un nouveau système démocratique de l'enseignement supérieur. Par ailleurs, l'ouverture du pays au monde extérieur a accéléré les processus éducatifs d'européanisation. Afin d'accompagner ces changements, l'État a adopté plusieurs lois ("Éducation», 1991; "Éducation supérieure », 2002) qui ont précisé la nouvelle orientation politique de l'enseignement supérieur. Plusieurs modifications, considérées comme progressistes, ont permis de faire évoluer sensiblement le système ukrainien :

- le passage à un paradigme humaniste en éducation ;

- l'introduction de nouveaux standards éducatifs et le changement de contenus des programmes ;

- l'introduction de la structure universitaire en grade: le «spécialiste junior» et le «bachelor » ( $1^{\mathrm{er}}$ cycle $)$, et le « spécialiste » et le « master » $\left(2^{\mathrm{e}}\right.$ Repères cycle $) ;^{1}$

- l'introduction de l'évaluation externe de la qualité des formations au moyen de procédures d'accréditations et d'habilitations des établissements et des programmes par la commission d'accréditation d'État;

- l'élargissement de l'autonomie des établissements et la démocratisation de la vie universitaire, notamment en valorisant le rôle du conseil scientifique, des syndicats étudiants et l'élection des recteurs. 
2 En effet, depuis 2005, l'Ukraine participe au processus de Bologne. Des mesures importantes ont été prises afin d'améliorer la lisibilité, la flexibilité et l'efficacité du système: modification de la structure universitaire, organisation modulaire des enseignements, mise en place du système de transfert de crédits ECTS, etc. Cependant, la rénovation de l'enseignement supérieur s'élabore difficilement. Un climat politique et économique instable ainsi que l'insuffisance de financements, la dérégulation institutionnelle, la massification des publics étudiants et la corruption sont des facteurs qui, parmi d'autres, freinent les progrès désirés.

\section{Structure et organisation de l'enseignement supérieur}

Depuis les années 1990, l'abolition du monopole d'État dans le domaine de l'enseignement supérieur a entrainé la croissance et la diversification d'établissements, surtout dans le secteur privé. En 2008, il y avait, en Ukraine, 904 établissements d'enseignement supérieur, dont 351 de niveaux d'accréditation III-IV (universités, académies, instituts font partie de l'enseignement supérieur long) et 553 de niveaux I-II (collèges et écoles professionnelles et techniques font partie de l'enseignement supérieur court) ${ }^{2}$. C'est pendant la période de l'indépendance et de la transition vers l'économie de marché que le nombre d'établissements a plus que doublé: de 156 à 351 (niveaux III-IV). Cette augmentation importante est le fait des établissements privés : de 25 à 202 (tous niveaux confondus).

4 Pour répondre aux exigences de plus en plus fortes de qualité et d'efficacité, la tendance de transformation des instituts en universités se confirme ces dernières années. En effet, ces deux types d'établissements d'enseignement supérieur long existaient dans le système soviétique. La différence essentielle entre les deux résidait dans le contenu de l'enseignement : général et théorique pour les universités ; professionnel et appliqué pour les instituts. De plus, les universités relevaient du ministère de l'Éducation, tandis que les instituts dépendaient d'autres ministères, des entreprises et même de conseils locaux. Cette disparité institutionnelle persiste dans le système actuel: les établissements d'enseignement supérieur se trouvent sous l'autorité de 26 ministères différents. Cela conduit au doublement des formations entre des établissements voisins, à l'augmentation d'établissements privés avec peu d'étudiants (700 étudiants en moyenne) et à l'impossibilité de mettre en œuvre les réformes en cours. Le ministère de l'éducation s'efforce de changer la situation. Il a été décidé d'optimiser le système, pendant les années 2009-2010, en fusionnant des établissements et en créant des pôles universitaires régionaux, professionnels ou de recherche.

5 Actuellement, il existe en Ukraine 91 universités, dont la plupart se nomment «Université nationale». Ce statut spécifique est donné aux établissements qui se distinguent par leur formation académique et professionnelle de haut niveau, par leur participation à la construction démocratique de la société ukrainienne ou encore par leur rayonnement international. En outre, le fonctionnement de plusieurs établissements d'importance historique a été rétabli, c'est le cas de l'Université nationale Académie KyivMohyla et de l'Université nationale Académie d'Ostroh.

6 La structure interne des universités n'a pas beaucoup changé : chaque établissement se compose de facultés ayant un doyen à leur tête ; la faculté se subdivise en chaires dirigées par un professeur. D'importants changements ont été apportés à la gestion et au 
fonctionnement des établissements avec l'objectif de démocratiser la vie universitaire. Il existe par exemple dans chaque établissement une haute assemblée collégiale qui adopte le statut et le règlement intérieur, approuve le rapport annuel d'activités présenté par le recteur, le règlement sur le fonctionnement des syndicats étudiants, etc. En outre, après les élections internes, cette assemblée collégiale recommande à l'autorité de tutelle le candidat aux fonctions de chef d'établissement.

\section{L'accès à l'enseignement supérieur}

7 Avant 2008, pour être admis dans l'enseignement supérieur, le candidat devait obtenir le certificat de l'enseignement secondaire et réussir les concours d'entrée organisés par les établissements. Le nombre de places disponibles a augmenté considérablement ces dernières années, mais l'État ne finance plus que 40 à $42 \%$ des admissions. Actuellement, une année universitaire coûte en moyenne 5000 à 7000 hrivnya $^{3}$. Il faut rappeler que, pendant l'époque soviétique, les études supérieures étaient gratuites. Selon le ministère, lors du concours pour ces " places budgétaires », il y avait en moyenne en 2007, au niveau national, 3,8 candidats pour chaque place offerte.

Or, les études supérieures sont considérées par les Ukrainiens comme l'étape la plus importante de la vie de leurs enfants. Selon une enquête, 66 \% des Ukrainiens pensent qu'elles sont nécessaires pour leurs enfants, et $8 \%$ désirent qu'ils obtiennent un doctorat ${ }^{4}$. Chaque année, pour se préparer aux examens et concours, les jeunes désirant devenir étudiants révisent seuls ou avec l'aide de professeurs. Cette pratique est devenue un véritable marché pour les professeurs du secondaire et du supérieur. C'est au cours de cette période que les parents dépensent le plus leurs économies et certains sont obligés de s'endetter lourdement. Cependant, pour la population, cette préparation intensive et coûteuse ne peut à elle seule garantir le succès aux examens: selon une opinion répandue, l'entrée dans l'enseignement supérieur, surtout dans les facultés prestigieuses, n'est pas possible sans pot de vin. La corruption est devenue un fait si habituel dans l'éducation qu'elle semble difficile à éradiquer, malgré les efforts considérables du ministère au cours de ces dernières années.

Pour toutes ces raisons et afin de lutter contre la corruption dans l'enseignement supérieur, le gouvernement a expérimenté depuis 2004, au niveau national, une procédure d'évaluation externe indépendante en s'appuyant sur l'initiative de la fondation Soros «Renaissance». Un centre indépendant d'évaluation de la qualité de l'éducation a pris en charge la mise en place du processus, le développement de tests dans onze disciplines (à l'exception des langues vivantes), la formation des examinateurs (en majorité les enseignants du secondaire). La réforme s'est concrétisée en 2008, première année de l'évaluation externe obligatoire pour tous les candidats et de la suppression totale des examens d'entrée. Aujourd'hui, les établissements d'enseignement supérieur sélectionnent leurs étudiants en se référant aux certificats délivrés par le centre indépendant d'évaluation.

Évidemment, cette mesure ne pourra éradiquer totalement la corruption. Ainsi, il reste un accès à l'enseignement supérieur réservé à certaines catégories de candidats et où les abus sont encore possibles. En 2008, on a noté l'augmentation significative des candidats avec certificat médical permettant l'admission dans le supérieur hors procédure générale. En outre, pour des filières spécifiques, les établissements organisent des examens en langues vivantes ou encore des concours « créatifs» (domaines artistiques et culturels). 
De plus, de nombreuses critiques portent sur la qualité des tests élaborés par le centre et sur son monopole absolu dans l'organisation de l'évaluation extérieure. Néanmoins, l'Ukraine a grand besoin de réformes décisives pour établir un système équitable et transparent. Elle peut tirer parti de cette période de changements pour améliorer les conditions de travail des enseignants afin qu'ils ne soient pas «obligés » de faire des « appels » financiers aux parents. De plus, la société ukrainienne attend d'autres mesures fortes qui permettront de rétablir la valeur des connaissances et des compétences, longtemps remplacée par le principe du « qui paie gagne ».

\section{Les étudiants et le financement des études}

11 La progression des effectifs des étudiants inscrits dans les établissements d'enseignement supérieur est significative: de 455000 en 1998-1999 à 633700 en 2007-2008. Si, pour la même période, le nombre d'étudiants est en légère diminution dans les établissements d'enseignement supérieur court, ce sont les établissements d'enseignement supérieur long des niveaux III et IV qui attirent davantage d'étudiants (de 290100 à 491 200). Selon le rapport de l'UNESCO-CEPES (2003), l'Ukraine se place en tête des pays d'Europe centrale et orientale (juste après la Pologne) pour le nombre d'étudiants pour cent mille habitants : 4084 en Pologne, 3920 en Ukraine, 3274 pour la Fédération russe, 2927 en Hongrie. En outre, l'enseignement supérieur ukrainien devient de plus en plus attractif pour les étudiants étrangers, la majorité de ces derniers venant d'Asie et d'Afrique. Leur nombre augmente de $10 \%$ par an et approche 40000 en 2008. Ils apprécient des coûts d'études moins élevés et un climat de sécurité plus favorable que dans d'autres pays voisins, notamment la Russie. À l'inverse, la mobilité des étudiants ukrainiens reste faible. Il existe toujours des obstacles à la promotion de la mobilité : régime lourd des visas, absence de soutien financier de l'État et bas niveau de vie de la jeunesse.

Cependant, cette augmentation significative des effectifs ne peut être décrite comme une véritable démocratisation de l'enseignement supérieur en Ukraine. C'est sans doute la crise économique des années 1990 qui a poussé les établissements d'enseignement supérieur à ouvrir des places et des filières payantes afin de trouver des financements complémentaires. De plus, ce développement des marchés dans l'enseignement supérieur a été lié aussi à une demande accrue pour une formation supérieure longtemps réservée à l'élite du système soviétique. Toutefois, il faut admettre qu'aujourd'hui les chances réelles de faire des études supérieures ne sont pas égales. Ainsi, pour $67 \%$ de la population ukrainienne, l'accès à l'enseignement devient de plus en plus difficile à cause des problèmes financiers. ${ }^{5}$

Les textes officiels ukrainiens expriment la volonté politique d'un accès équitable à l'enseignement supérieur en proposant deux axes. Le premier prévoit la lutte contre la corruption, en développant des modalités plus objectives, démocratiques et lisibles pour les concours d'entrée. Le deuxième axe consiste à motiver des jeunes gens pour les études supérieures, notamment, par le soutien et l'accompagnement des enfants doués. Ainsi, en 2007, un accès prioritaire était réservé aux élèves qui avaient gagné les olympiades nationales (1 200 étudiants) ou à ceux qui ont obtenu des certificats avec félicitations ${ }^{6}$ pour leurs études secondaires $(28000)$. De plus, l'État garantit la promotion des minorités et des populations défavorisées: dans cette catégorie se trouvent les populations des régions agricoles, les orphelins, les enfants handicapés, les enfants de militaires, la population des régions sinistrées de Tchernobyl, etc. Ils étaient 30000 en 2007 (21 000 en 
2006), soit 4,7\% des nouveaux étudiants à pouvoir accéder ainsi à l'enseignement supérieur.

14 En outre, depuis 2002, l'État a mis en place un système de crédits pour les étudiants qui financent leurs études supérieures. Ce système de crédits est assez complexe car il dépend du budget alloué par l'État ou les régions chaque année. L'étudiant signe un contrat avec l'établissement où il compte suivre ses études. Il doit rembourser son prêt quinze ans après l'obtention du diplôme avec un taux annuel d'intéressement de $3 \%$. En 2007, 4600 étudiants ont bénéficié de cette offre plutôt libérale du gouvernement.

\section{Problèmes d'efficacité}

Un large consensus existe parmi les acteurs sur le fait que l'enseignement supérieur ukrainien doit accroître son efficacité. En effet, en 2001, la majorité des enseignants du supérieur (61\%) estimaient que l'enseignement supérieur soviétique était meilleur. Ils étaient deux tiers à se prononcer pour la nécessité de changements et de réformes (Kipen dir., 2001). Le constat est également négatif en matière de qualité des formations : la moitié des enseignants déplorent sa dégradation. Ils expliquent cela par la baisse du niveau d'éducation des jeunes sortant de l'enseignement secondaire, ainsi que par leur incapacité à utiliser les connaissances acquises à l'université dans les situations professionnelles. Il est intéressant d'analyser les perceptions des mêmes enseignants quant aux priorités des changements pour redresser la situation : pour 79,5\%, l'action prioritaire est de faciliter l'accès aux études supérieures aux jeunes « doués " issus des familles défavorisées. Les autres actions concernent le développement des compétences professionnelles des enseignants (56\%), l'optimisation du processus de formation (50,3\% ), la nécessité de changements paradigmatiques (humanisation, informatisation, orientations vers l'international), etc.

16 La même médiocrité de qualité est révélée dans les enquêtes du Centre Razumkov auprès de la population ukrainienne (2006). Cependant, il existe une légère amélioration dans ce constat : en 2002, ils étaient $42,3 \%$ à penser que la qualité des études est moyenne, 52,5\% en 2004 et $50,6 \%$ en 2006. Il ressort de ces enquêtes que, pour les Ukrainiens, les établissements d'État proposent des formations de meilleure qualité que celles des établissements privés : 43,2 \% pour les établissements d'État, et 12,5\% pour le privé.

17 La qualité des formations dépend en grande partie des compétences des enseignants du supérieur. Le contexte d'exercice de leurs missions essentielles - l'enseignement et la recherche - a changé depuis l'indépendance du pays. Sous-financées pendant de nombreuses années ${ }^{7}$, les universités ont vu leurs meilleurs professeurs et chercheurs partir pour des structures privées car l'État n'arrivait pas à payer leurs faibles salaires à temps. Ceux qui sont restés ont eu des difficultés à s'adapter aux nouveaux étudiants, hétérogènes par leurs niveaux scolaires, leurs motivations, mais aussi par leurs milieux sociaux. Les enseignants se sont donc retrouvés en première ligne de tous les problèmes de l'enseignement supérieur.

Dans son rapport de 2008, le ministère de l'Éducation et de la recherche constate le vieillissement des enseignants du supérieur. Les jeunes scientifiques mécontents de leurs conditions de travail préfèrent partir travailler à l'étranger ${ }^{8}$. Les salaires des professeurs restent bas: cela les oblige à faire des heures supplémentaires ou à enseigner dans plusieurs établissements. Dans ces conditions, il est difficile d'exiger d'eux leur 
investissement dans des activités de recherche et d'espérer la compétitivité scientifique des universités ukrainiennes. Selon le ministère, en 2007, seuls $24 \%$ des diplômés de l' aspirantura (l'école doctorale) ont soutenu leur thèse de candidat ès sciences.

De plus, l'enseignement supérieur ukrainien souffre de son académisme. Les formations restent peu adaptées au marché du travail. Lors de la mise en place du processus de Bologne, les problèmes de qualité, d'orientation, de poursuite d'études ne sont pas devenus des enjeux importants. Le décalage entre la politique volontariste du ministère et la morosité des acteurs de terrain n'a pas permis une réflexion approfondie sur ces questions.

\section{Bilan critique}

L'enseignement supérieur ukrainien reste encore très marqué par la culture éducative de l'époque soviétique. Confrontés à la crise économique des années 1990, les universités et les instituts se sont transformés en «entreprises » cherchant seuls leur financement de manière légale - et parfois illégale. Ils se sont adaptés à la massification et à la diversification de leurs publics et ont créé de nouvelles filières en multipliant les diplômes. Dans ces conditions, le bilan de l'évolution de l'enseignement supérieur en Ukraine est mitigé et contrasté. La décentralisation et l'ouverture du pays ont permis aux établissements de découvrir de nouvelles formes de fonctionnement, de faire des projets et de changer leur pédagogie. Mais, cette période de transformations et d'innovations marque aussi une crise d'identité, surtout pour les universités ukrainiennes «nationales». Quelle université veut-on construire? Avec quelles missions: la production de nouvelles connaissances, la transmission de la culture et des savoirs, ou la formation professionnelle des jeunes pour les besoins de l'économie ? Comment peut-elle devenir efficace dans un contexte d'austérité financière quasi permanente?

21 Il est difficile d'estimer les bénéfices du Processus de Bologne pour l'enseignement supérieur en Ukraine, car il existe toujours des problèmes d'égalité des chances et de qualité des formations. Cependant, cette réforme "européenne», inspirée par un autre événement national la "Révolution orange ", a provoqué l'enthousiasme et l'engagement des acteurs pour des changements. Un énorme progrès a été fait dans la refonte de la structure universitaire. Les deux premiers cycles (Bachelor et Master) sont déjà en place, et les réflexions continuent sur la modification des études doctorales. En effet, il existe toujours deux titres scientifiques (candidat ès sciences et docteur ès sciences) et il est possible de les obtenir non seulement dans les universités mais aussi dans les instituts de l'Académie des sciences, ou encore, de manière autonome, sous la direction d'un chercheur. À terme, il est prévu de garder seulement le titre scientifique de docteur mais la réorganisation du troisième cycle s'annonce difficile.

De même, un progrès rapide a été fait dans l'organisation modulaire des enseignements et dans la mise en place du système de crédits ECTS. Cependant, l'efficacité des études supérieures paraît fragile, et il reste beaucoup de choses à faire afin d'améliorer le processus éducatif. Pour le ministère de l'Éducation, il est urgent de développer des procédures fiables d'assurance qualité. Si les mesures externes d'assurance qualité (accréditation et habilitation d'établissements et de formations, système national d'évaluations et de classements d'établissements, commissions d'assurance qualité au niveau national et régional, publications des résultats) sont déjà en place, les mesures internes ne sont pas suffisamment développées par les établissements. Ainsi, les acteurs 
du système ukrainien cherchent les conseils d'autres pays qui ont réussi à introduire la culture et les valeurs de qualité au niveau des établissements.

Enfin, l'ambition des politiques ukrainiennes est de construire une "société de la connaissance ». Cela signifie la nécessité non seulement de changements structurels et institutionnels profonds mais aussi de l'investissement important de l'État dans la recherche. Malheureusement, il sera difficile, voire impossible, pour les universités ukrainiennes d'obtenir cet investissement dans les prochaines années.

\section{BIBLIOGRAPHIE}

KIPEN V. dir. (2001) : L'attitude des académiciens de l'enseignement supérieur de l'Ukraine envers les changements et les réformes, rapport de recherche, Fondation Renaissance et Université Nationale de Donetsk (en ukrainien).

Revue du centre ukrainien pour les études économiques et politiques Alexandre Razumkov « La sécurité nationale et la défense ", $n^{\circ}$ 11, 2006 (en ukrainien).

\section{NOTES}

1. La réforme des structures universitaires doit se terminer en 2010. Il est envisagé de garder les niveaux de bachelor (34 ans), de master (1-2 ans), de docteur en philosophie et de docteur ès sciences ( 3 ans).

2. Les chiffres utilisés dans cet article sont tirés du rapport du ministère de l'éducation et de la recherche de l'Ukraine «L'enseignement supérieur en Ukraine - le choix européen : l'état, les problèmes, les perspectives ", Osvita Ukraini, n 21-22 (905), 19 mars 2008 (en ukrainien).

3. Soit 1000 US \$. Le salaire minimum mensuel ne dépasse pas $150 \$$ en Ukraine.

4. Lubinets K. (2006) «Les particularités de la mise en œuvre des projets internationaux en Ukraine : le cas des projets de la Banque mondiale » [Pisliadiplomna osvita $v$ Ukraini], $\mathrm{n}^{\circ}$ 1, pp. 8-14 (en ukrainien).

5. Lubinets K. (2006), ibid.

6. À la fin des études secondaires, les élèves reçoivent un certificat (atestat) qui peut être accompagné d'une médaille d'or ou d'argent qui correspond à la mention «très bien » avec les félicitations.

7. Le sous-financement de l'enseignement supérieur en Ukraine est estimé de 60 à $70 \%$. Source : Danylichin B., Kutsenko V. (2005) «Le modèle d'innovation du développement économique : rôle de l'enseignement supérieur », Visnik NAN Ukraini, n 9, pp. 26-35.

8. 10 à $12 \%$ de nouveaux diplômés partent travailler à l'étranger chaque année (Source : ibid.). 
INDEX

Mots-clés : enseignement supérieur, réforme

Index géographique : Ukraine

\section{AUTEUR}

\section{MARINA SACILOTTO-VAYLENKO}

Marina Sacilotto-Vasylenko est chercheur postdoctoral au CREF à l'Université Paris Ouest Nanterre. Ses travaux, s'inscrivant dans le champ de l'éducation comparée, portent sur la formation et le développement professionnel des enseignants. Elle est membre du conseil du réseau européen Teacher Education Policies in Europe (TEPE) ainsi que du réseau européen Research Partnerships in Education de l'European Educational Research Association (EERA). 